Résumés des conférences et travaux

\title{
Histoire et archéologie des mondes musulmans
}

Jean-Michel Mouton

\section{OpenEdition \\ Journals}

\section{Édition électronique}

URL : https://journals.openedition.org/ashp/3528

DOI : $10.4000 /$ ashp.3528

ISSN : 1969-6310

Éditeur

Publications de l'École Pratique des Hautes Études

\section{Édition imprimée}

Date de publication : 1 septembre 2020

Pagination : 60-63

ISSN : 0766-0677

\section{Référence électronique}

Jean-Michel Mouton, « Histoire et archéologie des mondes musulmans », Annuaire de l'École pratique des hautes études (EPHE), Section des sciences historiques et philologiques [En ligne], 151 | 2020, mis en ligne le 09 juillet 2020, consulté le 06 juillet 2021. URL : http://journals.openedition.org/ashp/3528 ; DOI : https://doi.org/10.4000/ashp.3528 


\title{
HISTOIRE ET ARCHÉOLOGIE DES MONDES MUSULMANS
}

\author{
Directeur d'études : M. Jean-Michel Mouton
}

Programme de l'année 2018-2019 : I. La Syrie aux XII ${ }^{e}$-XIII siècles. - II. Études de la collection papyrologique des « Papiers de Damas » : les requêtes.

\section{La notion de frontière dans le monde musulman médiéval}

Les conférences de la première partie de cette année ont été consacrées tout d'abord à la notion de frontière dans le monde musulman médiéval, puis plus particulièrement, entre les États francs et les principautés musulmanes de Syrie aux XII ${ }^{\mathrm{e}}$ et $\mathrm{XIII}^{\mathrm{e}}$ siècles et enfin, à une échelle plus précise, au nord du royaume de Jérusalem, entre la seigneurie de Sidon et la principauté de Damas, en liaison avec la fouille archéologique conduite dans ce secteur.

Il apparaît clairement que la perception de la frontière dans le monde musulman médiéval n'est pas précise et est bien souvent multiforme. Si la perception d'entités politiques distinctes est très nette, par exemple entre états croisés et états musulmans, les confins de ceux-ci sont multiples comme le montre clairement le voyage effectué en 1183 par le voyageur andalou Ibn Ğubayr entre Damas et Acre. La frontière c'est à la fois une borne sur un chemin, un poste de douane, une forteresse-frontière. La particularité de l'époque des croisades est de dessiner des lignes de fortifications qui peuvent apparaître comme des lignes-frontières, mais qui s'effacent peu à peu au $\mathrm{XIII}^{\mathrm{e}}$ siècle avec le démantèlement de certaines fortifications par les Ayyoubides et ensuite par les Mamlouks le long de la côte syrienne.

Un autre aspect de la frontière apparaît dans les nombreux traités conclus au $\mathrm{XIII}^{\mathrm{e}}$ siècle entre croisés et Mamlouks où le partage des territoires se fait par casaux ou villages dont les limites sont parfaitement connues des habitants. On pourrait dans l'absolu, à partir de ces limites, dessiner sur une carte, une ligne frontière entre états croisés et états francs, mais elle n'exprimerait aucune réalité vécue par les habitants de ces contrées. Cette complexité et ce chevauchement des « frontières » ont amené certains chercheurs à nier sa réalité dans la perception médiévale de l'espace, mais bien au contraire, ce sont les formes multiples de ces zones de séparation qu'il faut sans doute retenir.

Dans la seconde partie de l'année, les conférences ont été consacrées à certains aspects de la vie du sultan Saladin (1138-1193). Les épouses, les enfants et la famille du sultan, les résidences royales et les loisirs de Saladin ont donné lieu à une première série de conférences. Les autres ont porté sur les modes de gouvernance de Saladin, sur la façon de gérer tout un Empire en étudiant notamment les modes de diffusion des instructions et des informations sur un vaste espace et les relations du sultan avec les membres de sa famille, les émirs, les ambassadeurs, etc. Les présents et les dons volontaires (șadaqa) sont un des instruments majeurs de cette gouvernance. 
Les documents étudiés durant la deuxième heure de la conférence proviennent, comme les années précédentes, de la collection des « Papiers de Damas » découverte lors de l'incendie de la grande mosquée de Damas en 1893 et transférée à Istanbul en 1911. Ces documents photographiés dans les années 1960 par Dominique et Janine Sourdel ont déjà donné lieu à plusieurs publications, portant notamment sur les actes notariés. L'attention cette année a été portée plus particulièrement sur des requêtes adressées à des militaires par une clientèle très variée constituée tant d'hommes de religions que de gestionnaires de leurs iqta $\bar{a}^{\prime}$-s. Un document particulièrement intéressant et en liaison directe avec la première partie du cours est une requête des habitants du petit village de Bayt Ahūn (aujourd'hui Bayt Yāḥūn au Sud-Liban) alors sous domination franque qui demandent au sultan, sans doute Saladin, à entrer sur le territoire du sultan à Bānyās pour y trouver refuge. On a là un nouveau témoignage de ces migrations villageoises vers la Syrie qui étaient jusque-là surtout connues par la famille des Banū Qudāma venue s'installer à Damas depuis le village de Ğammā'il relevant de la seigneurie franque de Naplouse. Les Banū Qudāma trouvèrent d'abord refuge dans l'oratoire d'Abū Șāliḥ à Bāb Šarqī, la porte orientale de Damas, avant de fonder le faubourg de Șālihiiyya sur les premières pentes du Qāsyūn.

\section{Fouilles de la forteresse d'Abū l-Hasan (Liban)}

Après une campagne préparatoire de 5 jours en 2017, une deuxième campagne de terrain de 13 jours en 2018 sur le site, une troisième campagne de trois semaines a eu lieu sur le site de la forteresse médiévale d'Abū l-Hasan au Liban en mai 2019 avec le soutien de la commission des fouilles archéologiques du ministère de l'Europe et des Affaires étrangères.

La forteresse d'Abu 1-Hasan occupe un site de méandre dans la vallée du nahr Awwalī à une vingtaine de kilomètres à l'est de Sidon sur les premières pentes du mont Liban. Elle est située sur un éperon rocheux qui domine de plus de 100 mètres le fleuve qui l'enserre. On accède à la forteresse par une rampe d'accès, tracée dans la boucle du méandre. Elle est défendue par une première tour carrée qui est suivie d'un passage entre des murs guidant l'approche vers une seconde tour-porte bien conservée en élévation. Passée la tour-porte, une autre porte donne accès à un couloir avec des escaliers permettant de monter sur la terrasse supérieure où se développe la forteresse. Le mur de ce couloir est percé d'archères vers l'extérieur. Ce couloir se trouve lui-même battu depuis le niveau sommital par une série d'archères. Les principales structures de la forteresse sont situées sur la plate-forme sommitale du promontoire rocheux large d'une trentaine de mètres et longue de 150 mètres. Un escalier taillé dans l'à-pic du rocher à l'opposé de l'entrée a été découvert cette année, il permettait de mettre directement en contact la forteresse avec le fleuve et pouvait servir aussi d'issue pour l'évacuation de la garnison en cas d'assaut.

Cette forteresse, alors qualifiée de «presidium», est mentionnée pour la première fois dans les chroniques en 1128 lorsque les « brigands » qui l'occupaient sont assiégés par le patriarche de Jérusalem Gormond de Picquigny, puis par le roi de Jérusalem Baudouin II qui la conquiert et l'intègre au royaume latin. Cependant la chronologie du site, jusque-là établie à partir des sources littéraires, a été totalement 
bouleversée à la suite de la campagne de 2019. La découverte de structures et d'artefacts (monnaies et céramiques) d'époque hellénistique et romaine montre la présence ancienne d'un habitat perché que l'on rencontre dans d'autres régions du Liban à la même époque. La construction du « presidium » correspond sans doute une refondation du site au $\mathrm{X}^{\mathrm{e}}$ siècle, c'est-à-dire à la fin de l'époque abbasside ou au début de la période fatimide.

L'occupation franque à partir de 1128 se traduit par la construction d'une véritable forteresse occupée durant plus d'un demi-siècle par les chrétiens. Elle est conquise en 1187 par Saladin après la bataille de Hattin. Les émirs ayyoubides qui se succèdent alors comme gouverneurs de la forteresse ont pour principale mission de l'intégrer à la ligne de fortifications qui protège le sud-ouest de la principauté de Damas, mais la $V^{e}$ croisade et la prise de Damiette entraîne en 1218 ou 1219 le démantèlement, sur ordre du prince de Damas, de toutes les forteresses musulmanes à l'ouest du Jourdain au nombre desquelles doit se trouver Abū 1-Hasan. Le site est alors abandonné et son territoire, au gré des traités de paix entre Francs et musulmans, revient à la famille Grenier, seigneurs de Sidon, puis aux Templiers. Abū 1-Ḥasan est reconquise par les Mamlouks à la fin du XIII' ${ }^{\mathrm{e}}$ siècle.

Cela ne signifie sans doute pas l'abandon définitif du site. Un abbé du monastère voisin du Saint-Sauveur recueillit en effet au début du $\mathrm{xx}^{\mathrm{e}}$ siècle des témoignages oraux qui gardaient le souvenir d'une restauration et d'une brève réoccupation du site sous l'émir Yousef Chéhab dans les années 1770 grâce à un financement obtenu auprès des marchands français et italiens de Sidon. Les seules traces archéologiques repérées à ce jour de cette dernière phase d'occupation se limitent à la présence de quelques tessons d'époque ottomane.

Les problématiques de cette recherche portent tout d'abord sur le rôle de la forteresse comme place-frontière entre le royaume latin de Jérusalem et la principauté musulmane de Damas. Il s'agit ainsi d'évaluer la place stratégique qu'elle occupait sur la route Sidon-Damas permettant le franchissement du mont Liban, ainsi que sur la ligne de fortifications située à l'ouest du Jourdain. À l'échelle de la forteresse, il s'agit aussi de préciser la chronologie des différentes constructions pour analyser les apports successifs des occupants francs et musulmans et d'évaluer l'impact des nombreux tremblements de terre du XII ${ }^{\mathrm{e}}$ siècle sur les structures de l'édifice et les phases de restauration.

Le site avait jusque-là fait l'objet d'une brève notice descriptive dressée dans les années 1930 par Paul Deschamps. Les deux premières missions, en 2017 et 2018, ont été consacrées à la réalisation d'une orthophotographie du site au moyen d'un drone, complétée d'un levé topographique au tachéomètre afin de dresser le plan des structures visibles de la forteresse et le relief en courbes de niveau. Nous avons également relevé et étudié deux des portes du système d'entrée et les élévations du front est, au-dessus de la plate-forme sommitale. Enfin une fouille a été conduite dans une petite salle de la plate-forme sommitale afin d'évaluer le potentiel stratigraphique du site.

Durant la campagne 2019, deux secteurs de fouilles ont été ouverts. Le premier a été celui de la tour-porte I implantée sur la rampe d'accès à la forteresse au sud de l'éperon rocheux. Cette structure de forme trapézoïdale $(8 \times 8,30 \mathrm{~m})$ est pourvue de deux portes sur sa face nord et d'une batterie de trois archères à simple ébrasement 
sur sa face sud. Le chemin d'accès à la forteresse la traverse décrivant un virage à $180^{\circ}$ à l'intérieur de la tour. On y accède par la porte sud-est précédée d'un escalier aux larges et profondes marches aménagées pour les chevaux. Le sol intérieur de la tour utilise le rocher taillé de gradins très approximatifs permettant de franchir la déclivité entre les deux portes de la tour. Le passage, à l'intérieur de la tour, est bordé au nord et au sud de deux banquettes profondes et hautes d'environ un mètre. Au-delà d'une fonction de repos pour les gardiens et de contrôle de la circulation des personnes et des bêtes traversant la tour-porte, la banquette sud, qui présente deux niveaux, desservait les trois archères. Cet aménagement très voisin dans sa conception de celui découvert par notre équipe à Șadr au Sinaï pourrait dater du dernier tiers du XII ${ }^{\mathrm{e}}$ siècle au moment de la reconquête de la forteresse par Saladin. Les piédroits de la porte nord-est de la tour à laquelle aboutit l'escalier ont entièrement disparu et ont fait l'objet d'une récupération qui pourrait correspondre à la phase de démantèlement de la forteresse en 1218-1219. L'essentiel du mobilier trouvé durant la fouille de ce secteur est constitué de carreaux d'arbalète, de pointes de flèche, de clous ainsi que d'une marelle.

Le second secteur de fouille est celui de la partie sud de la salle 5 qui est la salle la plus vaste $(17 \times 4 \mathrm{~m})$, située sur la partie sommitale. Il s'agissait, à travers la fouille d'un espace central, de déterminer les différentes phases d'occupation de la forteresse. Les cinq phases d'occupation qui ont pu être mises en évidence modifient profondément la chronologie de la forteresse établie jusque-là. Ainsi une première phase d'occupation antique a pu être mise en évidence. À ces structures abandonnées à la fin de l'Antiquité succède une phase de construction (phase II), sans doute celle $\mathrm{du}$ «presidium» mentionné par Guillaume de Tyr. Les analyses ${ }^{14} \mathrm{C}$ permettent de la situer dans le courant du $\mathrm{x}^{\mathrm{e}}$ siècle. La phase III correspond à la fortification de la plate-forme sommitale avec la construction des murs fortifiés est et sud de la salle 5 . Ces travaux doivent sans doute être attribués aux Francs qui font la conquête du site en 1128. La phase IV, qui correspond à la clôture de la salle 5, a livré de la céramique des $\mathrm{XII}^{\mathrm{e}}-\mathrm{XIII}^{\mathrm{e}} \mathrm{s}$. Cette datation concorderait avec l'occupation ayyoubide du site après la conquête musulmane de 1187. La dernière phase (phase V) n'a pas livré d'éléments de datation. Elle pourrait correspondre à la réoccupation tardive d'époque ottomane. Il faut ajouter qu'un très abondant matériel (céramiques, armes, os et objets en os) a été découvert dans cette salle. 